Submitted to the Annals of Applied Statistics

\title{
A BAYESIAN MODEL AVERAGING APPROACH FOR OBSERVATIONAL GENE EXPRESSION STUDIES - SUPPLEMENTAL MATERIAL
}

\author{
By Xi Kathy Zhou*, Fei LiU ${ }^{\dagger}$, And Andrew J. DAnnenberG* \\ *Weill Medical College of Cornell University \\ ${ }^{\dagger}$ IBM Watson Research Center
}

\section{Simulation Study.}

1.1. Simulation of microarray data. In the simulation study, we assumed complex correlations among covariates as described in the article. The proportions of subjects in different groups defined by the categories of the covariates are shown in Table 1.

TABLE 1

Proportions of subjects in different groups defined by the categories of the three covariates, i.e., smoking status, gender and heavy drinking status. All three covariates are binary variables with categories denoted by either 1 or 0.

\begin{tabular}{lrrrrrrrr}
\hline Groups & $(0,0,0)$ & $(1,0,0)$ & $(0,1,0)$ & $(1,1,0)$ & $(0,0,1)$ & $(1,0,1)$ & $(0,1,1)$ & $(1,1,1)$ \\
\hline Proportion (\%) & 7.5 & 32.5 & 5 & 5 & 5 & 5 & 32.5 & 7.5 \\
\hline
\end{tabular}

In the following, we describe the procedures for simulating one microarray data set based on model

$$
y_{i j}=\beta_{1 j} s_{i}+\beta_{2 j} g_{i}+\beta_{3 j} d_{i}+\varepsilon_{i j},
$$

where $\varepsilon_{i j} \sim \mathrm{N}\left(0, \sigma_{j}^{2}\right)$.

- For each gene $j, j=1, \ldots, m$, a gene specific variance is drawn based on

$$
\sigma_{j}^{2} \sim \frac{d_{0} s_{0}^{2}}{\chi_{d_{0}}^{2}}
$$

where $\chi_{d_{0}}^{2}$ is a $\chi^{2}$ - distribution with $d_{0}$ degrees of freedom. Both $d_{0}$ and $s_{0}$ are tuning parameters that can be used to control the amount of the gene-wise variances.

- The value of an indicator of differential expression with respect to covariate $k$ for gene $j, D_{k j}$ is drawn randomly from a binomial distribution

$$
D_{k j} \sim \operatorname{Binom}\left(f_{k}\right)
$$


TABLE 2

Power of different methods for identifying genes differentially expressed between smokers and non-smokers at $5 \%$ FDR under different simulation settings.

\begin{tabular}{|c|c|c|c|c|c|c|c|c|c|c|c|c|c|c|}
\hline$\overline{f_{s}}$ & $f_{g}$ & $f_{d}$ & $S M_{1}$ & $S M_{2}$ & $S V A$ & $B M A_{1}$ & $B M A_{1} *$ & $\begin{array}{l}B M A_{1} \\
(c=3)\end{array}$ & $\begin{array}{r}B M A_{1} * \\
(c=3)\end{array}$ & $\begin{array}{l}B M A_{1} \\
(c=5)\end{array}$ & $\begin{array}{r}B M A_{1} * \\
(c=5)\end{array}$ & $B M A_{2}$ & $B M A_{3}$ & $M M$ \\
\hline \multicolumn{15}{|c|}{$n=40$} \\
\hline 0.10 & 0.05 & 0 & 139 & 96 & 83 & 145 & - & 142 & - & 143 & - & 119 & 149 & 155 \\
\hline 0.10 & 0.05 & 0.05 & 126 & 80 & 72 & 137 & 131 & 134 & 132 & 132 & 132 & 124 & 137 & 150 \\
\hline 0.05 & 0.10 & 0 & 42 & 31 & 31 & 51 & - & 49 & - & 50 & - & 46 & 52 & 52 \\
\hline $\begin{array}{c}0.05 \\
n\end{array}$ & $\begin{aligned} & 0.10 \\
= & 80\end{aligned}$ & 0.10 & 46 & 30 & 26 & 56 & 52 & 52 & 51 & 51 & 50 & 49 & 57 & 58 \\
\hline 0.10 & 0.05 & 0 & 286 & 294 & 290 & 346 & - & 341 & - & 340 & - & 335 & 344 & 356 \\
\hline 0.10 & 0.05 & 0.05 & 239 & 250 & 248 & 317 & 297 & 318 & 291 & 318 & 289 & 308 & 318 & 334 \\
\hline 0.05 & 0.10 & 0 & 94 & 113 & 110 & 147 & - & 146 & - & 146 & - & 135 & 146 & 152 \\
\hline 0.05 & 0.10 & 0.10 & 82 & 108 & 106 & 145 & 122 & 142 & 116 & 140 & 114 & 138 & 142 & 147 \\
\hline
\end{tabular}

where $f_{k}$ is the proportion of genes that are differentially expressed with respect to covariate $k$.

- The value of parameter $\beta_{k j}$ is generated randomly as follows

$$
\beta_{k j} \sim\left\{\begin{array}{cc}
0 & \text { if } D_{k j}=0 \\
\mathrm{~N}\left(0, v_{0} \sigma_{j}^{2}\right) & \text { if } D_{k j} \neq 0
\end{array}\right.
$$

where $v_{0}$ is the tuning parameter that controls the variation of the non-zero $\beta_{k j}$.

- The expression level of gene $j$ for subject $i, y_{i j}$ can then be generated using following

$$
y_{i j} \sim \mathrm{N}\left(\beta_{1 j} s_{i}+\beta_{2 j} g_{i}+\beta_{3 j} d_{i}, \sigma_{j}^{2}\right) .
$$

In our simulation, the values of $d_{0}, s_{0}$ and $v_{0}$ are fixed at 1000, 2 and 0.5, respectively.

1.2. Sensitivity analysis of the BMA approach. The sensitivity of performance of the BMA approaches to the choice of cut-off $c$ and to the mis-specification of model space was examined. The gene ranking performance was shown in Fig 1 (a) and (b), and Table 2. We can see that the BMA approach with empirical prior is relatively robust with respect to the value of $c . B M A_{1}$ appeared to be slightly better than $B M A_{1}(c=3)$ and $B M A_{1}(c=5)$ in Fig 1(b). As expected, when important covariate $d$ is excluded from the model space $\left(B M A_{1} *\right)$, there is a decrease in ranking performance but $B M A_{1} *$ still performs better than all the single model approaches. In terms of $F D R$ estimation accuracy (Fig 1 (c) and (d)), we can see both the value of $c$ and the omission of important covariate in model space can notably affect the accuracy of peFDR. $B M A_{1}$ again appeared to be a more robust choice. 


\section{Application.}

2.1. Microarray study of airway epithelium samples. The Venn diagram (Fig 2) showed large disagreement among top smoking related genes identified by different approaches.

\section{References.}

Leek, J. T., Storey, J. D. (2007). Capturing heterogeneity in gene expression studies by surrogate variable analysis. PLoS Genetics, 3(9):1724-1735. 


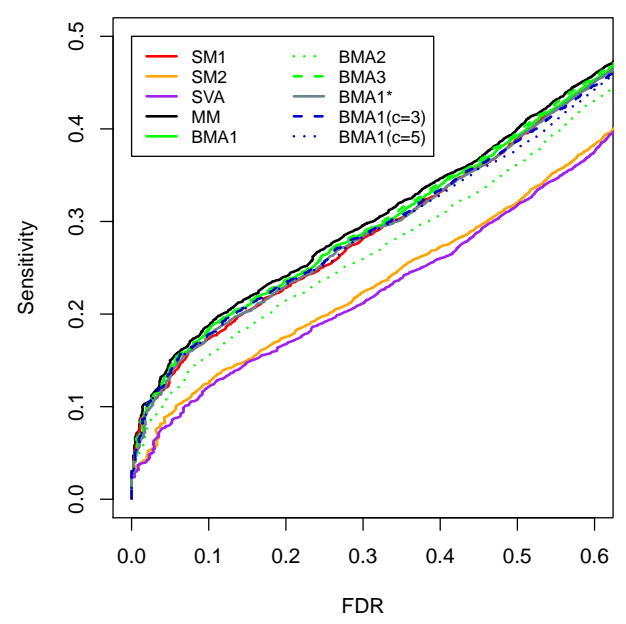

(a). $f_{s}=0.1, f_{g}=0.05, f_{d}=0, n=40$.

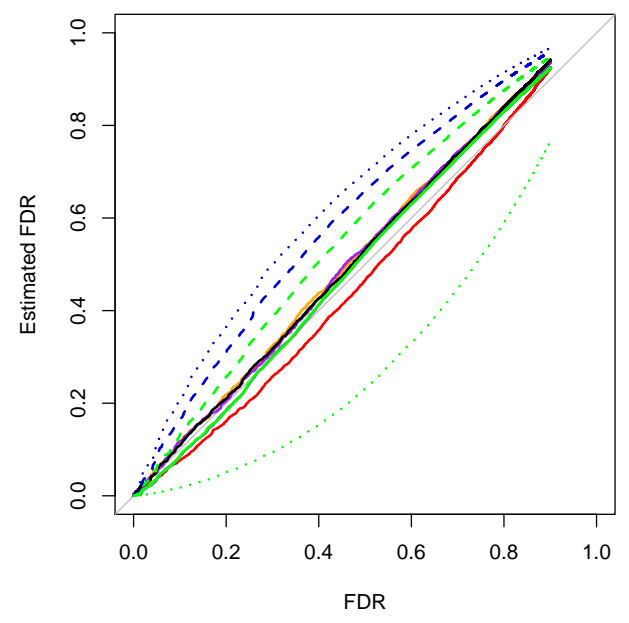

(c). $f_{s}=0.1, f_{g}=0.05, f_{d}=0, n=40$.

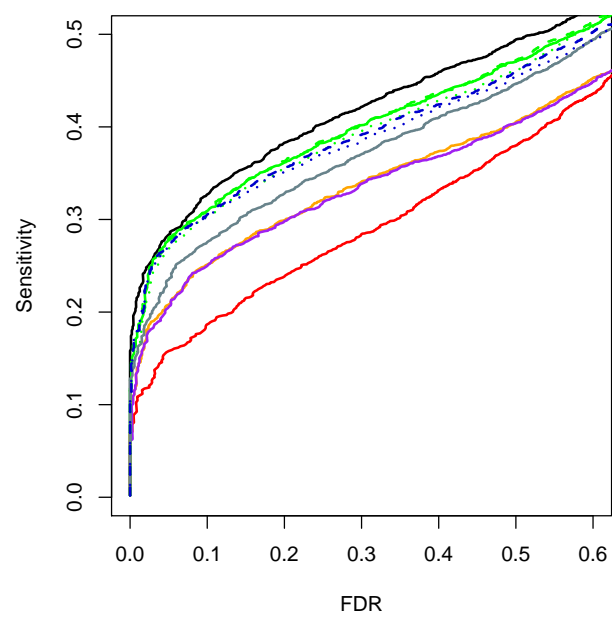

(b). $f_{s}=0.05, f_{g}=0.1, f_{d}=0.1, n=80$.

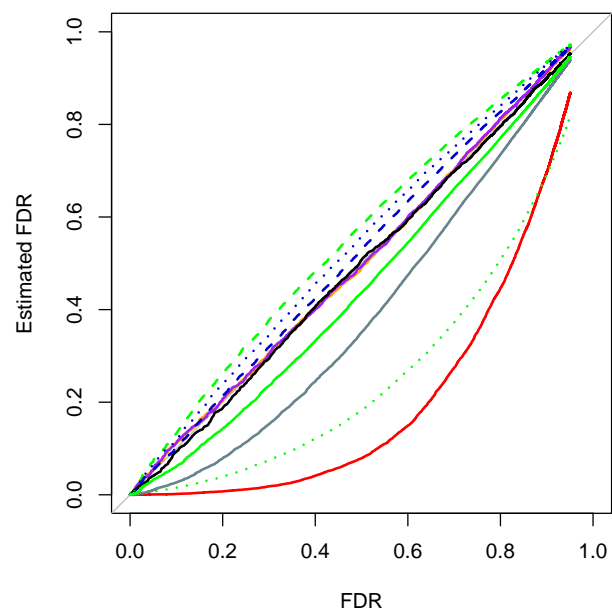

(d). $f_{s}=0.05, f_{g}=0.1, f_{d}=0.1, n=80$.

Fig 1: Sensitivity vs. FDR curves ((a) and (b)) and estimated FDR vs. true FDR $((c)$ and $(d))$ in two simulation settings. The approaches are: the single model approaches without covariate adjustment $\left(S M_{1}\right)$ and with covariate adjustment $\left(S M_{2}\right)$, the surrogate variable approach $(S V A)$, the multi-model approach $(M M)$, the BMA approach with the empirical prior where $c=1$ $\left(B M A_{1}\right)$, the BMA approach with uniform prior $\left(B M A_{2}\right)$, the BMA approach with the true proportion of $\mathrm{DE}$ genes as the prior $\left(B M A_{3}\right)$, the BMA approach with empirical prior under $c=1$ but without an important covariate in the model space $\left(B M A_{1} *\right)$, and the BMA approach with empirical prior under $c=3$ and $c=5\left(B M A_{1}(c=3)\right.$ and $B M A_{1}(c=5)$, respectively). 


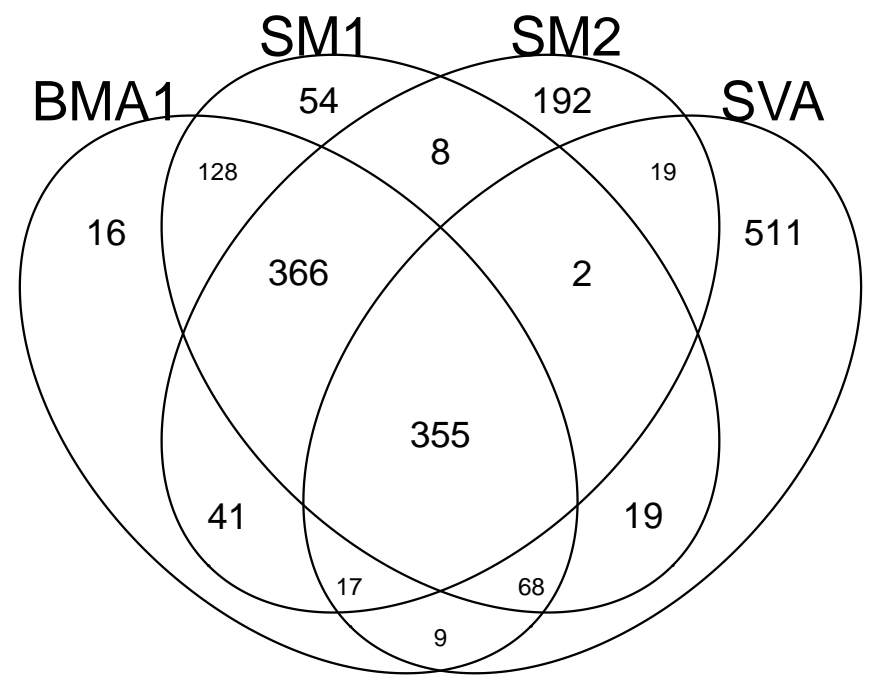

Fig 2: Venn diagram of smoking-related genes ranked within top 1000 by any of the four approaches: the Bayesian model averaging approach $\left(B M A_{1}\right)$, the single model approach with no covariate adjustment $\left(S M_{1}\right)$, the single model approach with adjustment for all recorded covariates $\left(S M_{2}\right)$ and the surrogate variable approach $(S V A)$. 